\title{
Alternating current magnetic susceptibility of a molecular magnet submonolayer directly patterned onto a micro superconducting quantum interference device
}

\author{
M. J. Martínez-Pérez, ${ }^{1,2}$ E. Bellido, ${ }^{3}$ R. de Miguel, ${ }^{4}$ J. Sesé, ${ }^{2,4}$ A. Lostao, ${ }^{4,5}$ \\ C. Gómez-Moreno, ${ }^{4,6}$ D. Drung, ${ }^{7}$ T. Schurig, ${ }^{7}$ D. Ruiz-Molina, ${ }^{3, a)}$ and F. Luis ${ }^{1,2, b)}$ \\ ${ }^{1}$ Instituto de Ciencia de Materiales de Aragón (ICMA), CSIC-Universidad de Zaragoza, \\ Pedro Cerbuna 12, E-50009 Zaragoza, Spain \\ ${ }^{2}$ Dpto. de Física de la Materia Condensada, Universidad de Zaragoza, \\ Pedro Cerbuna 12, E-50009 Zaragoza, Spain \\ ${ }^{3}$ Centro de Investigación en Nanociencia y Nanotecnología (CIN2, CSIC-ICN) Edificio CM7, \\ Esfera UAB, Campus UAB, E-08193 Cerdanyola del Vallés, Spain \\ ${ }^{4}$ Laboratorio de Microscopías Avanzadas (LMA)—Instituto de Nanociencia de Aragón (INA), \\ Universidad de Zaragoza, 50018 Zaragoza, Spain \\ ${ }^{5}$ Fundación ARAID, E-50004, Aragón, Spain \\ ${ }^{6}$ Dpto. de Bioquímica, Universidad de Zaragoza, Pedro Cerbuna 12, E-50009 Zaragoza, Spain \\ ${ }^{7}$ Physikalisch-Technische Bundesanstalt (PTB) Abbestraße 2-12, D-10587 Berlin, Germany
}

(Received 14 March 2011; accepted 16 June 2011; published online 20 July 2011)

We report the controlled integration, via dip pen nanolithography, of monolayer dots of ferritin-based $\mathrm{CoO}$ nanoparticles $\left(12 \mu_{\mathrm{B}}\right)$ into the most sensitive areas of a microSQUID sensor. The nearly optimum flux coupling between these nanomagnets and the microSQUID improves the achievable sensitivity by a factor $10^{2}$, enabling us to measure the linear susceptibility of the molecular array down to very low temperatures $(13 \mathrm{mK})$. This method opens the possibility of applying ac susceptibility experiments to characterize two-dimensional arrays of single molecule magnets within a wide range of temperatures and frequencies. (C) 2011 American Institute of Physics. [doi:10.1063/1.3609859]

The ac magnetic susceptibility of magnetic nanoparticles and single molecule magnets (SMMs) provides useful information on their spin and magnetic anisotropy, ${ }^{1}$ as well as on the magnetic relaxation mechanisms. ${ }^{2-4}$ Miniaturized superconducting quantum interference devices (SQUIDs) ${ }^{5-9}$ should eventually become capable ${ }^{8,10}$ of measuring the magnetization reversal of a SMM ( $\mu_{\mathrm{i}} \sim 20 \mu_{\mathrm{B}}$ for the archetypal $\mathrm{Mn}_{12}$ molecule). However, detecting the linear response sets even more stringent conditions: at $T=1 \mathrm{~K}$, a magnetic field $H=24 \mathrm{~A} / \mathrm{m}(0.3 \mathrm{Oe})$ induces a magnetic polarization $\langle\mu\rangle \simeq 0.007 \mu_{\mathrm{B}}$ on the same $\mathrm{Mn}_{12}$ cluster. Measuring the susceptibility of even a molecular monolayer represents therefore a considerable challenge, which requires to take the sensitivity of magnetic susceptometry beyond its actual limits. ${ }^{5}$ To maximize the magnetic coupling between SMMs and the SQUID, molecular nanomagnets need to be deposited onto specific areas of the sensor. ${ }^{8,10}$ Even though diverse techniques have been developed for structuring molecules and nanoparticles on sensors, ${ }^{9,11,12}$ such a controlled integration remains extremely challenging.

In the present work, we apply dip pen nanolithography $(\mathrm{DPN})^{13}$ to deposit monolayer dots of ferritin-based nanomagnets on the most sensitive areas of a microSQUID ac susceptometer. With its direct write capabilities, DPN is an attractive tool for the nanostructuration on surfaces and for controlling the number of units deposited. ${ }^{14-17}$ The sample consisted of cobalt oxide nanoparticles, $\simeq 2 \mathrm{~nm}$ in diameter,

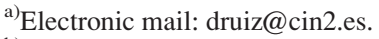

b)Electronic mail: fluis@unizar.es.
}

whose magnetic moment $\simeq 12 \mu_{\mathrm{B}}$ is close to that of typical SMMs. ${ }^{4}$ These particles (CoO@Apoferritin) are synthesized inside the protein nanocavity of horse spleen apoferritin ${ }^{18}$ and can be patterned and immobilized over different substrates. ${ }^{19}$ The bulk magnetic susceptibility of this material was characterized using $\sim 10^{-9} \mathrm{Kg}$ of $\mathrm{CoO} @$ Apoferritin. Further details of this and other experimental aspects are given in the Supplementary material (see Ref. 20).

The microSQUID susceptometer used for these studies has been described elsewhere. ${ }^{20-22}$ The pick-up coil most sensitive ("active") areas were identified by calculating (see Fig. 1(c) and Ref. 20) the magnetic flux $\Phi_{\text {coupled }}$ generated by a sample located at a particular position. The coupling can be quantified by a flux coupling factor

$$
\alpha=\frac{\Phi_{\text {coupled }}}{\mu_{\mathrm{i}}} \frac{B_{\mathrm{p}}}{i_{\mathrm{p}}},
$$

where $\mu_{\mathrm{i}}$ is the magnetic moment induced by the excitation magnetic field $B_{\mathrm{p}}$ and $i_{\mathrm{p}}$ is the electrical current circulating via the primary coil. We find that $\alpha$ can be enhanced by more than three orders of magnitude by simply placing the nanomagnets sufficiently close to the coil wire edges, where the magnetic field lines concentrate.

The rational deposition of CoO@Apoferritin on these active areas is depicted in Fig. 2. Three rows of CoO@Apoferritin dots separated by $4 \mu \mathrm{m}$ were fabricated on the pickup coils labeled 3 and 4 in Fig. 1(a) by traversing the tip soaked with the ferritin-based nanoparticles over the specific areas. The first row was deposited on the primary $\mathrm{Nb}$ coil, and the other two were deposited on the $\mathrm{SiO}_{2}$ layer. The 


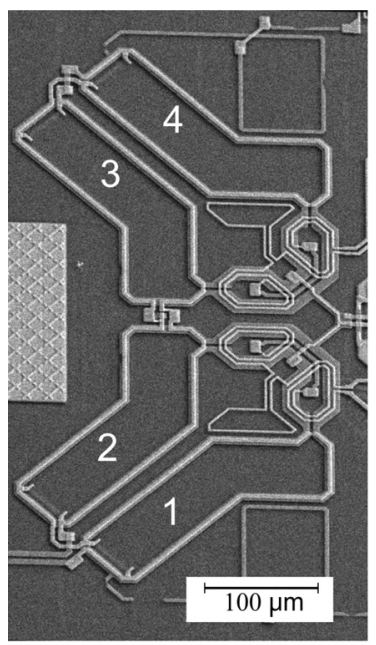

(a)
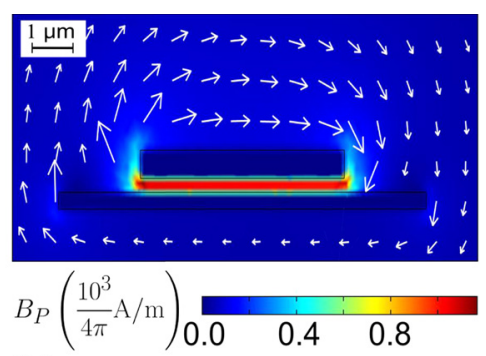

(b)

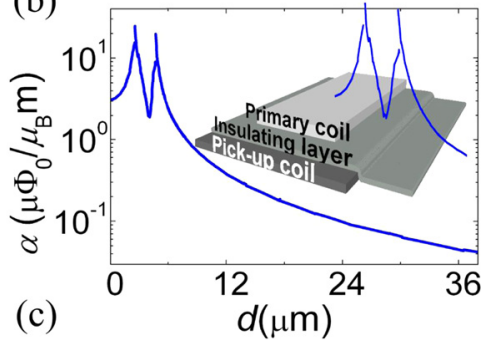

FIG. 1. (Color online) (a) SEM image of the SQUID showing the four rectangular shaped pick-up coils with effective areas of $63 \mu \mathrm{m} \times 250 \mu \mathrm{m}$. (b) Finite element calculation of the excitation magnetic field $\left(B_{\mathrm{p}}\right)$ created by a $i_{\mathrm{p}}=500 \mu \mathrm{A}$ current flowing through the primary coil, approximated by a circular spire. (c) Numerical calculations of $\alpha$ as a function of the distance from the center of the pick-up coil wire towards the center of the coil, approximated also by a circle. The inset shows a 3-D cross section of the pick-up and primary coil wires, where the $\alpha$ profile has been superimposed.

SEM images (Fig. 2(b)) reveal the high precision achieved in positioning the dots at the positions of maximum $\alpha$. The dots dimensions were measured by AFM (see Figs. 2) on arrays deposited on bare $\mathrm{SiO}_{2}$ and $\mathrm{Nb}$ substrates under identical conditions. We find average diameters of $1.3 \mu \mathrm{m} \pm 0.1 \mu \mathrm{m}$ and $1.8 \mu \mathrm{m} \pm 0.1 \mu \mathrm{m}$ for $\mathrm{SiO}_{2}$ and $\mathrm{Nb}$ substrates, respectively. The average dot height was $11 \mathrm{~nm} \pm 1 \mathrm{~nm}$ in both, close to the size of a single protein (ca. $12 \mathrm{~nm}$ ), thus showing that each dot is a monolayer. According to these values, the average number of CoO@Apoferritin units per dot is $10^{4}$ and $2 \times 10^{4}$ for $\mathrm{SiO}_{2}$ and $\mathrm{Nb}$, respectively. The number of

(a)

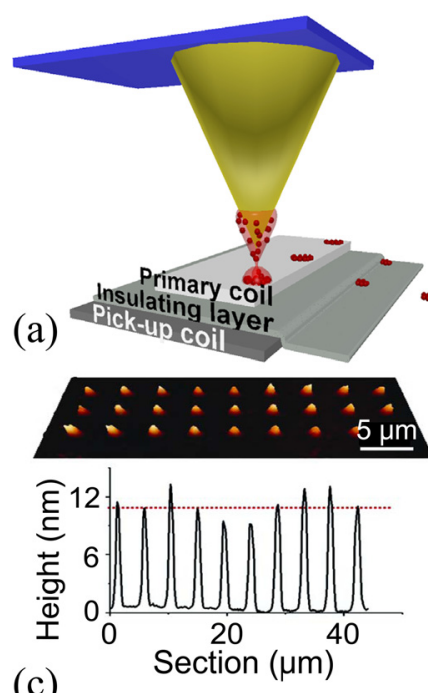

(c)
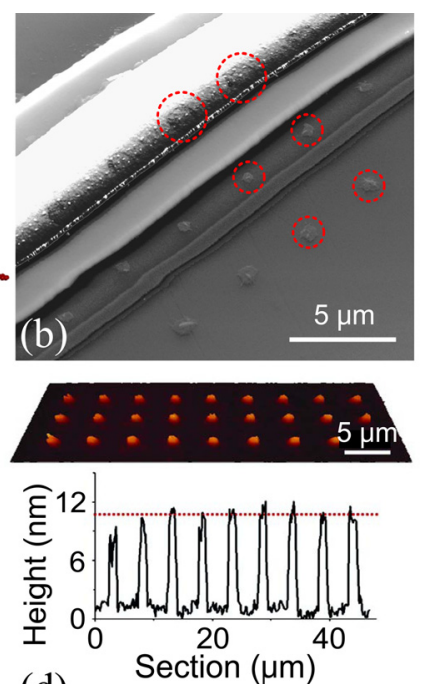

(d)
CoO@Apoferritin units deposited over the pick-up coils is $n \sim 10^{7}$.

The large coupling between the CoO@Apoferritin dots and the SQUID enabled us to measure their magnetic susceptibility down to $T=13 \mathrm{mK}$ (Fig. 3). Below $400 \mathrm{mK}$, a temperature dependent signal shows up above the background signal of the bare sensor that was previously characterized. ${ }^{22}$ Furthermore this signal shows the same qualitative dependence on temperature as the susceptibility $\chi^{\prime}$ of a bulk-like sample of CoO@Apoferritin measured with the same sensor under the same conditions. ${ }^{20}$ The magnetic polarization of the array can be estimated as $\langle\mu\rangle=n \chi^{\prime} B_{\mathrm{p}}$. Its maximum value, at $T \simeq 50 \mathrm{mK}$, amounts to only $2.3 \times 10^{5} \mu_{\mathrm{B}}$.

Below approximately $100 \mathrm{mK}, \chi^{\prime}$ depends on frequency. This shows the existence of a thermally activated spin reversal with characteristic timescale $\tau=\tau_{0} \exp \left(U / k_{\mathrm{B}} T\right)$, where $\tau_{0}$ is an attempt time and $U$ is the activation energy of the reversal process. ${ }^{23,24}$ When $\tau$ becomes comparable to $1 / \omega$, the spins cannot follow in phase the oscillations of the excitation magnetic field. The maximum of $\chi^{\prime}$ vs $T$ that we observe for the array (see Fig. 3(a) defines the "blocking" temperature, characteristic of a SMM, which occurs when $\tau \gtrsim 1 / \omega$. $^{2,4}$ Curiously enough, the bulk $\chi^{\prime}$ shows no clear maxima above $13 \mathrm{mK}$. At first, this might suggest that the blocking temperature, thus also $U$, is enhanced in the array by the interaction of the molecules with the substrate. Alternatively, the temperature shift can be ascribed to a different thermalization of both samples. In the array, with its larger contact-area to volume ratio, the molecules can properly thermalize with the surrounding He bath. In contrast, the actual temperature of the bulk sample can stay above that of the He bath (and thermometer), therefore not reaching the blocking temperature. This interpretation is supported by the fact that the bulk susceptibility is shifted with respect to that of the array already at $T \lesssim 200 \mathrm{mK}$, when $\chi^{\prime}$ is nearly independent of frequency and therefore relaxation mechanisms should not influence its temperature dependence. ${ }^{20}$

Using these data it is possible to determine the average coupling factor $\alpha$. For this, we replace in Eq. (1) $\mu_{\mathrm{i}}$ by the net polarization of the molecular array $\langle\mu\rangle$, defined above. The

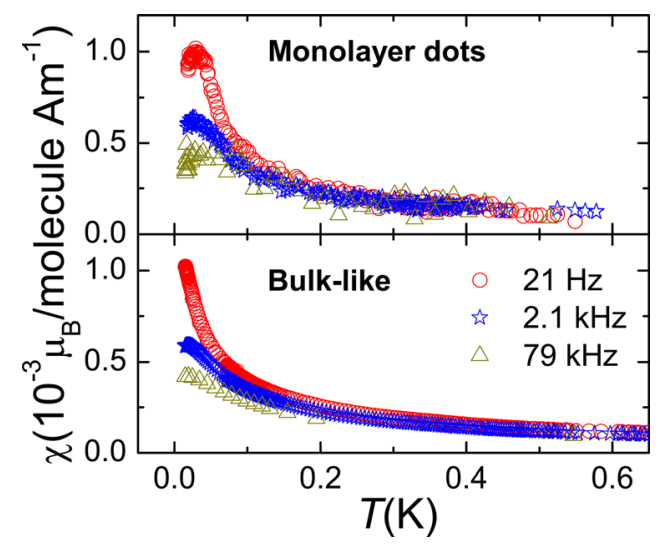

FIG. 3. (Color online) Top: in-phase ac magnetic susceptibility of $10^{7}$ CoO@Apoferritin molecules arranged as a (sub)monolayer. The outof-phase component lies below the sensitivity limits of detection and it is therefore not shown. Bottom: in-phase susceptibility of $\sim 10^{-9} \mathrm{Kg}$ of CoO@Apoferritin ( $10^{12}$ units). 
experimental $\Phi_{\text {coupled }}$ can be determined from the SQUID's output voltage, since they are related trough fabrication parameters. Inserting real values in Eq. (1) gives $\alpha=28.6( \pm 0.1) \mu \Phi_{0} / \mu_{\mathrm{B}} \mathrm{m}$, of the same order of magnitude, albeit more than three times larger, as the average $\alpha=8.0( \pm 0.1) \mu \Phi_{0} / \mu_{\mathrm{B}} \mathrm{m}$ extracted from the numerical calculations shown in Fig. $1 .^{20}$ The discrepancy can be ascribed to the approximations made to simplify these calculations, in particular the use of circular primary and pick-up coils. This parameter gives a spin sensitivity $\simeq 300 \mu_{\mathrm{B}} \mathrm{Hz}^{-1 / 2}$ at $13 \mathrm{mK}$, which represents an enhancement of two orders of magnitude with respect to the previous calibration performed with a $45 \mu$ m thick $\mathrm{Pb}$ sphere. ${ }^{22}$

Summarizing, we have fabricated submonolayer arrays of ferritin-based nanomagnets $\left(12 \mu_{\mathrm{B}}\right)$ on those regions that have a maximum flux coupling with a microSQUID loop. This controlled integration enhances the sensitivity by a factor $10^{2}$. Furthermore, the molecular deposition is carried out under ambient temperature and pressure conditions and implies no chemical functionalization of the sensor neither of the sample. The enhanced sensitivity has enabled us to directly measure the linear susceptibility of the molecular array, which shows that each molecule preserves its magnetic properties. The present technology opens the possibility of using the ac susceptibility to characterize two-dimensional arrays of single-molecule magnets. The same approach can be also applied to optimize the flux coupling of magnetic molecules to any other superconducting circuit, such as planar resonators, therefore contributing to the realization of hybrid architectures for quantum computation. ${ }^{25}$

This work was partly funded by Grant No. MAT200913977-C03 of the Spanish MICINN and the ConsoliderIngenio project on Molecular Nanoscience. M.J.M.-P. thanks CSIC for a JAE predoctoral fellowship. E.B. and R.dM. thank the MICINN for FPI and FPU predoctoral grants. A.L. thanks ARAID for financial support. We acknowledge helpful discussions with C. Carbonera and D. Maspoch. M.J.M.-P. and E.B. contributed equally to the present work.
${ }^{1}$ F. Luis, F. Bartolomé, F. Petroff, J. Bartolomé, L. M. García, C. Deranlot, H. Jaffrès, M. J. Martínez, P. Bencok, F. Wilhelm, A. Rogalev, and N. B. Brookes, Europhys. Lett. 76, 142 (2006).

${ }^{2}$ J. M. Hernández, X. X. Zhang, F. Luis, J. Bartolomé, J. Tejada, and R. Ziolo, Europhys. Lett. 35, 301 (1996).

${ }^{3}$ E. M. Chudnovsky and J. Tejada, Macroscopic Quantum Tunneling of the Magnetic Moment, Cambridge Studies in Magnetism (Cambridge University Press, Cambridge, 1998).

${ }^{4}$ D. Gatteschi, R. Sessoli, and J. Villain, Molecular Nanomagnets (Oxford University Press, Oxford, 2006).

${ }^{5}$ D. D. Awschalom, J. R. Rozen, M. B. Ketchen, W. J. Gallagher, A. W. Kleinsasser, R. L. Sandstrom, and B. Bumble, Appl. Phys. Lett. 53, 2108 (1988).

${ }^{6}$ W. Wernsdorfer, K. Hasselbach, D. Mailly, B. Barbara, A. Benoit, L. Thomas, and G. Suran, J. Magn. Magn. Mater. 145, 33 (1995).

${ }^{7}$ S. K. H. Lam and D. L. Tilbrook, Appl. Phys. Lett. 82, 1078 (2003).

${ }^{8}$ J. P. Cleuziou, W. Wernsdorfer, V. Bouchiat, T. Ondarcuhu, and M. Monthioux, Nat. Nanotech. 1, 53 (2006).

${ }^{9}$ L. Hao, C. Amann, J. C. Gallop, D. Cox, F. Ruede, O. Kazakova, P. Josephs-Franks, D. Drung, and Th. Schurig, Appl. Phys. Lett. 98, 092504 (2011).

${ }^{10}$ V. Bouchiat, Supercond. Sci. Technol. 22, 064002 (2009).

${ }^{11}$ S. K. H. Lam, W. Yang, H. T. R. Wiogo, and C. P. Foley, Nanotechnology 19, 285303 (2008).

${ }^{12}$ L. Bogani, C. Danieli, E. Biavardi, N. Bendiab, A. L. Barra, E. Dalcanale, W. Wernsdorfer, and A. Cornia, Angew. Chem. Int. Ed. 48, 746 (2009).

${ }^{13}$ R. D. Piner, J. Zhu, F. Xu, S. Hong, C. A. Mirkin, Science 283, 661 (1999).

${ }^{14}$ S. Sekula, J. Fuchs, S. Weg-Remers, P. Nagel, S. Schuppler, J. Fragala, N. Theilacker, M. Franzreb, C. Wingren, P. Ellmark, C. A. K. Borrebaeck, C. A. Mirkin, H. Fuchs, and S. Lenhert, Small 4, 1785 (2008).

${ }^{15}$ K. Salaita, Y. Wang, and C. A. Mirkin, Nat. Nanotech. 2, 145 (2007).

${ }^{16}$ B. Basnar and I. Willner, Small 5, 28 (2009).

${ }^{17}$ E. Bellido, R. de Miguel, D. Ruiz-Molina, A. Lostao, and D. Maspoch, Adv. Mater. 22, 352 (2010).

${ }^{18}$ M. Uchida, S. Kang, C. Reichhardt, K. Harlen, and T. Douglas, Biochim. Biophys. Acta 1800(8), 834 (2010).

${ }^{19}$ E. Bellido, R. de Miguel, J. Sesé, D. Ruiz-Molina, A. Lostao, and D. Maspoch, Scanning 32, 35 (2010).

${ }^{20}$ See supplementary material at http://dx.doi.org/10.1063/1.3609859 for a detailed description of the calculation of the SQUID-sample flux coupling factor and the sample characterization.

${ }^{21}$ M. J. Martínez-Pérez, J. Sesé, F. Luis, D. Drung, and T. Schurig, Rev. Sci. Instrum. 81, 016108 (2010).

${ }^{22}$ M. J. Martínez-Pérez, J. Sesé, F. Luis, R. Córdoba, D. Drung, T. Schurig, E. Bellido, R. de Miguel, C. Gómez-Moreno, A. Lostao, and D. RuizMolina, IEEE Trans. Appl. Supercond. 21, 345 (2011).

${ }^{23}$ L. Néel, Ann. Geophys. 5, 99 (1949).

${ }^{24}$ W. F. Brown, Phys. Rev. B 130, 1677 (1963).

${ }^{25}$ A. Imamoglu, Phys. Rev. Lett. 102, 083602 (2009). 


\section{SUPPLEMENTAL INFORMATION \\ for}

Ac magnetic susceptibility of a molecular magnet submonolayer directly patterned onto a micro superconducting quantum interference device

M. J. Martínez-Pérez, ${ }^{1,2}$ E. Bellido, ${ }^{3}$ R. de Miguel, ${ }^{4}$ J. Sesé,,${ }^{4,2}$ A. Lostao,${ }^{4,5}$ C. Gómez-Moreno, ${ }^{4,6}$ D. Drung, ${ }^{7}$ T. Schurig, ${ }^{7}$ D. Ruiz-Molina, ${ }^{3, *}$ and F. Luis ${ }^{1,2, \dagger}$ ${ }^{1}$ Instituto de Ciencia de Materiales de Aragón (ICMA), CSIC-Universidad de Zaragoza, Pedro Cerbuna 12, E-50009 Zaragoza, Spain

${ }^{2}$ Dpto. de Física de la Materia Condensada, Universidad de Zaragoza, Pedro Cerbuna 12, E-50009 Zaragoza, Spain

${ }^{3}$ Centro de Investigación en Nanociencia y Nanotecnología (CIN2, CSIC-ICN) Edifcio CM7, Esfera UAB, Campus UAB, E-08193 Cerdanyola del Vallés, Spain

${ }^{4}$ Instituto de Nanociencia de Aragón. Universidad de Zaragoza Edifcio I+D. Campus Río Ebro, E-50018 Zaragoza, Spain

${ }^{5}$ Fundación ARAID, Aragón, Spain

${ }^{6}$ Dpto. de Bioquímica, Universidad de Zaragoza, Pedro Cerbuna 12, E-50009 Zaragoza, Spain

${ }^{7}$ Physikalisch-Technische Bundesanstalt (PTB) Abbestra_e 2-12, D-10587 Berlin, Germany (Dated: June 14, 2011) 


\section{S1. Description of the $\mu$ SQUID}

A commercial easy-to-use two-stage SQUID sensor from Magnicon ${ }^{[1]}$ was selected since it is well suited to operate at $\mathrm{mK}$ temperatures with a broad bandwidth and sensitivities close to the quantum limit. We profited from our ability of modifying superconducting circuits ${ }^{[2]}$ to reroute some of the SQUIDs input wires to fabricate an integrated SQUID-susceptometer. These sensors are installed inside the mixing chamber of a Leiden Cryogenics dilution refrigerator providing a friendly tool capable of ac susceptibility measurements under extreme conditions of frequencies $(1 \mathrm{mHz}-1 \mathrm{MHz})$ and temperatures $(T \approx 13 \mathrm{mK})$. The sensor chosen for this experiment consists of four one-turn primary coils connected in series that create the exciting magnetic field and four one-turn pick-up coils connected in parallel that couple the sample response directly to the front-end dc SQUID. No flux transformer is needed since the pick-up coils are already part of the SQUID loop. The resulting signal is amplified by a 16-SQUID series array and the whole system works in Flux Locked Loop mode (FLL). Apart from a small background, the primary coils couple no net flux to the SQUID due to their gradiometric design, unless a magnetic sample is placed onto one of them. Such background signal can be easily compensated electronically or subtracted from the sample signal. These coils are rectangular shaped with approximate dimensions $63 \mu \mathrm{m} \times$ $250 \mu \mathrm{m}$. The pick-up coil wire cross dimensions are $9 \mu \mathrm{m} \times 300 \mathrm{~nm}$. The latter is covered with a $250 \mathrm{~nm}$-thick layer of $\mathrm{SiO}_{2}$, and finally the primary coil lies on top having a $5 \mu \mathrm{m} \times$ $500 \mathrm{~nm}$ cross area.

\section{S2. Calculation and measurement of the coupling factor $\alpha$}

The coupling factor is defined as $\alpha=\frac{\phi_{\text {coupled }}}{\mu_{i}} \frac{B_{\mathrm{P}}}{i_{\mathrm{P}}}$ where $\phi_{\text {coupled }}$ is the coupled magnetic flux in the SQUID loop generated by a sample with magnetic moment $\mu_{i}$ located at a particular position with respect to the coils boundaries. This magnetic moment is induced by the excitation magnetic field $B_{\mathrm{p}}$, created by an electrical current, $i_{\mathrm{p}}$, circulating via the primary coil.

The calculation was performed using finite element method software (COMSOL). First $\frac{\phi_{\text {coupled }}}{\mu_{i}}$ was calculated using the reciprocity theorem. This theorem states that calculating the 
flux generated per unit of magnetic moment is equivalent to calculating the magnetic field, generated at the location of the magnetic moment, per unit of current circulating through the SQUID pick-up coil. Figure S1 shows the result, where a circular shaped pick-up coil was used, having the same area as the real SQUID loop. The calculations take into account the Meissner effect in the superconductor using appropriate boundary conditions in the magnetic vector potential as already described. ${ }^{[3]}$

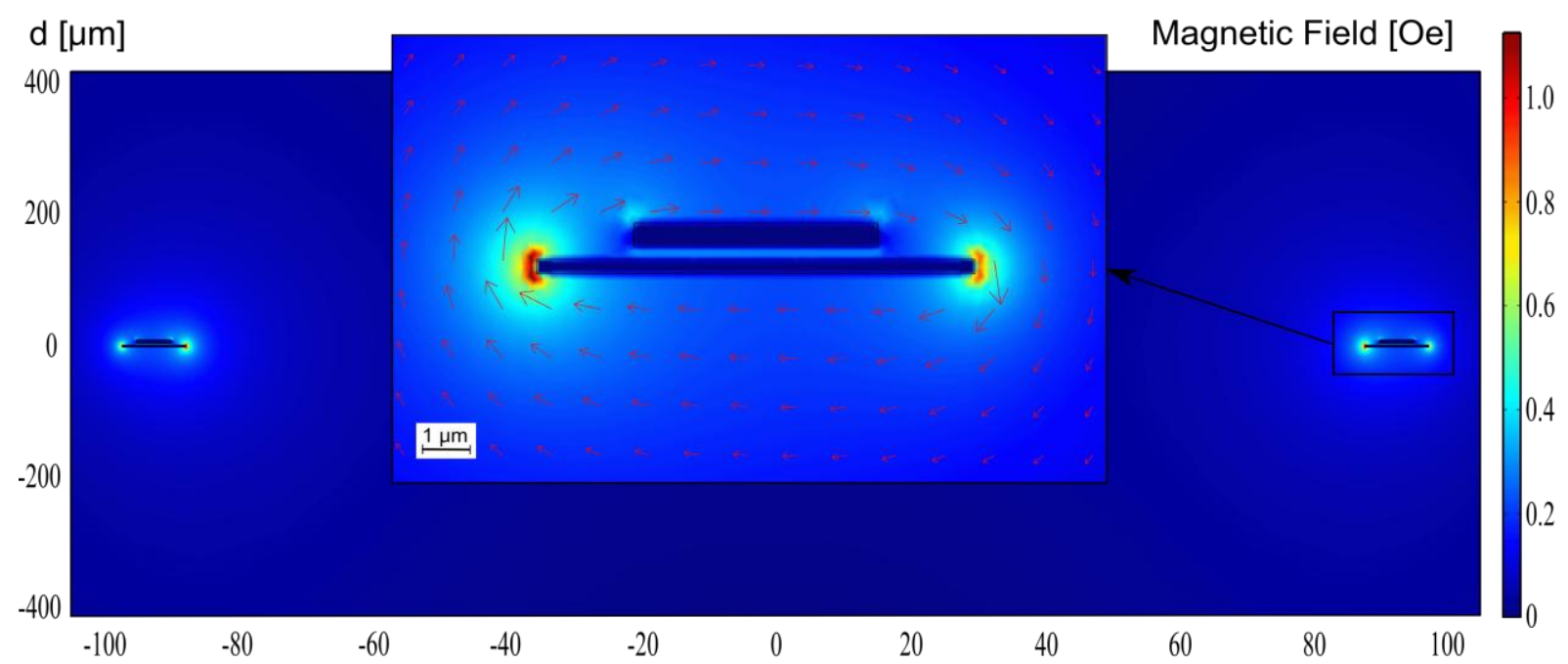

Figure S1. Finite element calculations of the magnetic field created by the pick-up coil with a driving current of $500 \mu \mathrm{A}$. The current circulating in the primary coil is forced to be zero.

Then $\frac{B_{\mathrm{P}}}{i_{\mathrm{P}}}$ is calculated using the same procedure (see Figure 1 in the manuscript), but taking into account that a shielding current in the pick-up coil will appear due to the flux conservation in the parallel gradiometer.

\section{S3. Preparation and characterization of the $\mathrm{CoO} @$ apoferritin}

CoO@apoferritin preparation. Horse spleen apoferritin (HsAFr) was purchased from Sigma-Aldrich. The apo form is obtained after controlled acidic treatment of the commercial sample to remove almost all the stored natural iron in the form of ferrihydrite. A few $\mathrm{Fe}^{3+}$ atoms anchored to internal nucleation centres are retained while maintaining intact a quaternary structure of the protein with molecular weight (MW) of $481 \mathrm{kDa}$. Cobalt oxide 
nanoparticles were synthesized following a previously reported method ${ }^{[4]}$ by introducing rational modifications that allowed us to achieve different results in cobalt loading and even composition of the nanoparticles. For these experiments, $500 \mathrm{~mL}$ of $100 \mathrm{mM} \mathrm{pH} 8.3 \mathrm{HEPES}$ buffer solution containing $0.5 \mathrm{mg} \cdot \mathrm{mL}^{-1} \mathrm{HsAFr}$ was prepared under argon atmosphere. The solution was stirred and ammonium cobalt sulfate hexahydrate (Sigma-Aldrich) and sodium sulphate were added to final concentrations of 3.0 and $37.5 \mathrm{mM}$ respectively, followed by the addition of hydrogen peroxide till a concentration of $1.5 \mathrm{mM}$. The solution was stirred for 20 minutes and then left at $50{ }^{\circ} \mathrm{C}$ overnight. A pool of enriched $2 \mathrm{~nm}$ cobalt oxide nanoparticles encapsulated ferritin was obtained. The syntheses did not generate any precipitate.

Transmission electron microscopy (TEM). The size and shape of the $\mathrm{CoO}$ nanoparticles formed inside the cavity of the ferritin protein were studied by TEM experiments. Measurements were performed using a Tecnai T20 thermo emission microscope at $200 \mathrm{kV}$. Samples were prepared by diluting and sonicating the ferritin-based cobalt oxide nanoparticles in water and deposited on a holey carbon 300 mesh copper grid (SPI Supplies) by placing the grid over a droplet of diluted sample for 2 minutes. Excess liquid was removed with a piece of "blotting paper" and the grid let to dry overnight.

The particle size distribution of the sample was obtained after a thorough analysis of the images obtained by TEM (Figure S2). The diameter of the nanoparticles ranges from 1.2 to $3.0 \mathrm{~nm}$, with a maximum number of particles (peak of the Gaussian curve) near $2 \mathrm{~nm}$. Moreover, as can be seen in Figure S3, most of $\mathrm{CoO}$ inside the nanoparticles is in the form of a unique particle with spherical-like shape. However, in a very limited number of cases with nanoparticle diameters over $3.0 \mathrm{~nm}$, (not-shown in the figure) bean-shaped or several small cores inside a single ferritin can be distinguished. This fact indicates that the in the case of the larger nanoparticles the growth takes place from two or three different nucleation centres placed on the inner cavity of the apoferritin. 


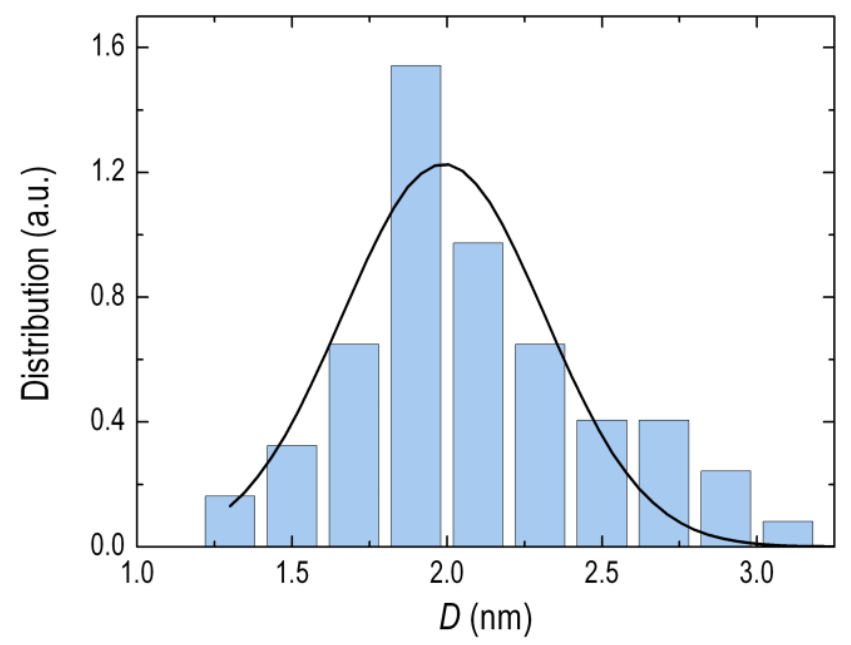

Figure S2. Particle size distribution of the $\mathrm{CoO} @$ apoferritin sample obtained from the analysis of the TEM images. The solid line is the Gaussian fit showing an average diameter of $D=2.0 \mathrm{~nm}$ and variance $\sigma=0.32$

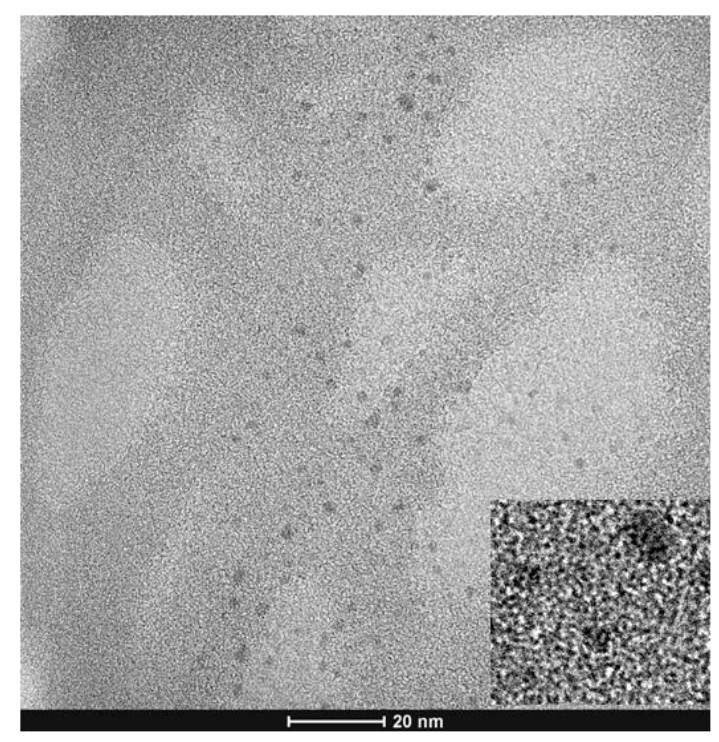

Figure S3. Typical TEM images of a CoO@apoferritin sample used in this work. The inset $(10 \times 21 \mathrm{~nm})$ is an enlarged picture showing the homogeneity in spherical shape of the prepared nanoparticles. Black spots represent the inorganic cores, whereas the protein shell is not visible.

Energy-dispersive X-ray spectroscopy (EDX). CoO@apoferritin nanoparticles were analyzed with a scanning electron microscope (SEM) Hitachi S-3400 N equipped with a Röntec XFlash de $\mathrm{Si}(\mathrm{Li}) \mathrm{EDX}$ analyzer. For this, the ferritin sample was dialyzed in Milli-Q water and lyophilized. A protein sample of the resulting powder was spread on carbon. Measurements were done using variable pressure at $60 \mathrm{~Pa}$, a $10 \mathrm{~mm}$ working distance and a tension in the filament of about $15 \mathrm{kV}$. Five different micrometric areas were analyzed by 
EDX giving an average percentage in cobalt of 1.30 over the whole powder sample. This is equivalent to 108 cobalt atoms per protein molecule.

$X$-ray photoelectron spectroscopy (XPS). The ferritin sample was dialyzed in Milli-Q water and lyophilized. Several milligrams were spread on a silicon substrate for the XPS surface analysis done with a KRATOS/AXIS Ultra DLD spectrometer. The C1s peak centred at 284.9 $\mathrm{eV}$ was used as a calibration reference. The values of all binding energies were shifted so as to keep the $\mathrm{C} 1 \mathrm{~s}$ binding energy constant.

Looking at the Co $2 p$ series, clear differences in the primary and satellite peak energies as well as the line shapes have been reported ${ }^{[5-6]}$ between metallic $\mathrm{Co}(0), \mathrm{Co}^{2+}$ in $\mathrm{CoO}$, and $\mathrm{Co}^{3+}$. These reference spectra provide useful fingerprints for determining the oxidation state of Co. A detailed comparison reveals that cobalt atoms in the CoO@apoferritin nanoparticles are clearly in the $2+$ formal oxidation state occupying octahedral sites (Figure S4). The superposition of the calculated sub-spectra agrees very well with the experimental results. Both the line shapes and binding energies corresponding to $\mathrm{Co}^{2+}$ match very well those measured on the nanoparticles. 


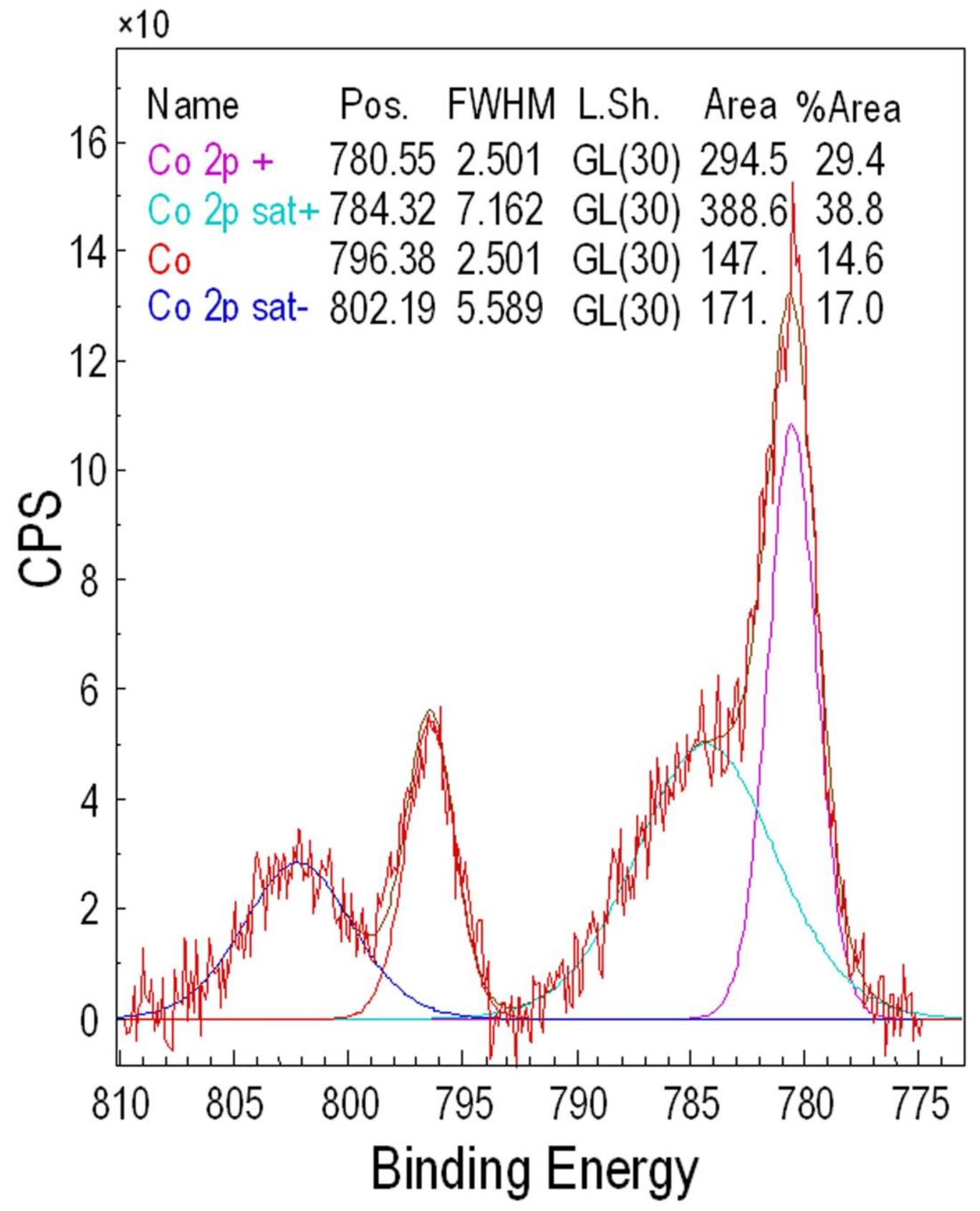

Figure S4. High-resolution Co $2 \mathrm{p}$ core-level spectrum obtained by XPS. The deconvolution into the different contributions corresponding to pure $\mathrm{Co}^{2+}$ asserts that the cobalt present in the NPs is in the $\mathrm{Co}^{2+}$ state.

\section{S4. Apoferritin stability}

To study the stability of the deposited apoferritin proteins exposed to vacuum and low temperature cycles fluorescence spectroscopy was used. Measurements were performed with a Perkin Elmer LS55 spectrofluorometer at $20^{\circ} \mathrm{C}$, using an excitation wavelength of $280 \mathrm{~nm}$. Initially, spectra of the bulk apoferritin in three different folding states in solution (native, a semi-denaturated state where the subunits retain their tridimensional structure and a completely unfolded state) were separately recorded to be used as models. Apoferritin was 
unfolded to a semi folded and unfolded states with $0.01 \mathrm{M}$ glycine hydrochloride buffer at $\mathrm{pH}$ 2.0 and $6 \mathrm{M}$ guanidine hydrochloride at $\mathrm{pH} 1.9$, respectively. The final spectra of the three different folding states are shown in Figure S5. The native protein showed a maximum emission at $323 \mathrm{~nm}$, a smaller peak at $340 \mathrm{~nm}$ and a shoulder at $360 \mathrm{~nm}$. In the semidenatured state, the maximum shifted towards $360 \mathrm{~nm}$, but the smaller peak remained at 340 $\mathrm{nm}$. The complete unfolding of the subunits further shifts the tryptophan emission to lower energy, presenting a maximum at $360 \mathrm{~nm}$, with a peak at $323 \mathrm{~nm}$, related to the fluorescence of tyrosyl residues, and a $340 \mathrm{~nm}$ shoulder.

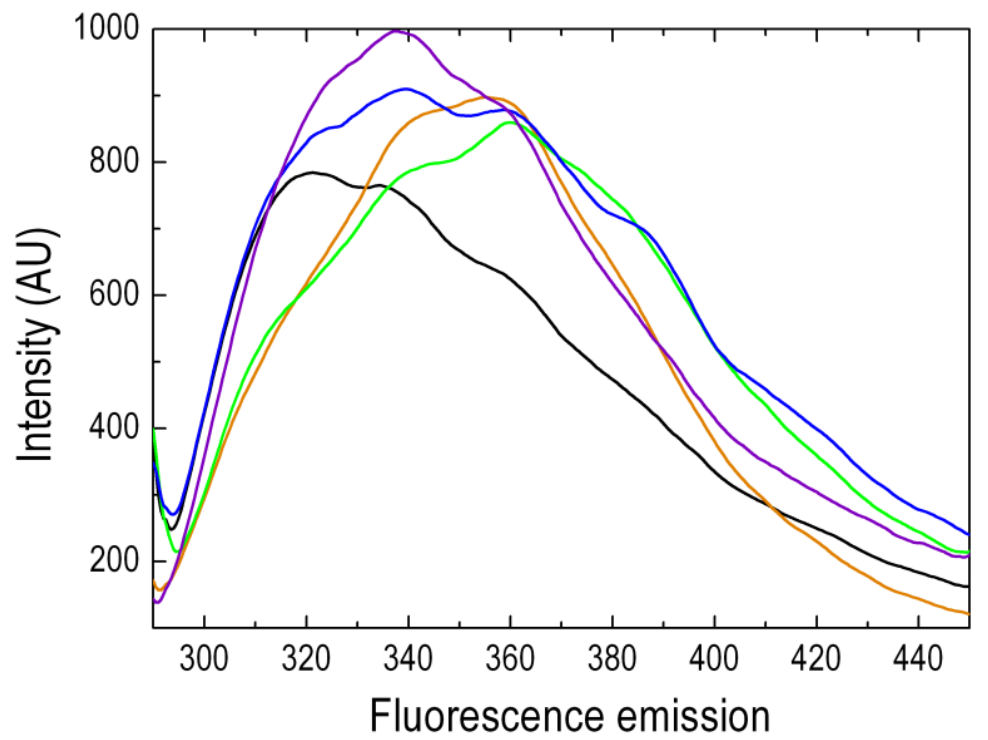

Figure S5. Fluorescence emission spectra of $0.25 \mathrm{mg} / \mathrm{mL} \mathrm{HsAFr}$ and their subunits in $10 \mathrm{mM}$ Tris-HCI buffer at pH 7.0. Excitation was at $280 \mathrm{~nm}$. (Blue line) bulk protein sample; (purple line) liquid protein sample obtain from dots deposited by DPN; (black line) native apoferritin; (orange line) monomeric subunits in $0.01 \mathrm{M}$ glycine hydrochloride buffer at $\mathrm{pH} 2.0$; (green line) apoferritin subunits in $6 \mathrm{M}$ guanidine hydrochloride at $\mathrm{pH} 1.9$.

Afterwards, experiments were performed on apoferritin samples deposited on $\mathrm{SiO}_{2} / \mathrm{Si}$ and $\mathrm{Nb}$ substrates, both as a bulk powder fixed with Apiezon grease or deposited from solution by drop-casting (the small amount of material deposited by DPN is below the threshold detection of our fluorescence experimental set-up). Such substrates were exposed to the same temperature cycles and vacuum conditions as the samples used for the magnetic measurements. However, due to experimental limitations that avoid direct in-situ fluorescence studies, the samples were removed from the surface before their study by extraction with water and posterior dilution in $10 \mathrm{mM}$ Tris- $\mathrm{HCl}$ buffer at $\mathrm{pH} 7.0$ down to a final concentration of $0.25 \mathrm{mg} / \mathrm{mL}$. In the case of the bulk sample the spectrum after removal 
showed emission maxima at $340 \mathrm{~nm}$ and smaller peaks at $323 \mathrm{~nm}$ and $360 \mathrm{~nm}$. The dipcoated sample exhibited maximum emission at $340 \mathrm{~nm}$, a smaller peak at $323 \mathrm{~nm}$ and a shoulder at $360 \mathrm{~nm}$. By comparison with the model spectra of the three folding states previously described, it is rather difficult to unequivocally assign the folding status of the complex quaternary protein structure in these samples. However, there is a clear tendency, according to their emission spectra, which indicates that both samples contain a mixture of states, where the protein is mostly folded or semi-folded.

\section{S5. Experimental details of the methodology for direct writing apoferritin/CoO@Apoferritin particles with DPN}

Apoferritin and $\mathrm{CoO} @$ Apoferritin sample preparation. The molecular ink was prepared by dissolving the lyophilized $\mathrm{CoO} @$ Apoferritin (concentration=55.2 $\mathrm{mg} \cdot \mathrm{mL}^{-1}$ ) in a phosphatebuffered saline (BupH PBS from Pierce) and glycerol (Sigma-Aldrich, $\geq 99.5 \%$, ACS grade) solution (80/20\%, respectively). The use of additives, such as glycerol, in the protein ink solution is a well-known strategy because it significantly enhances the protein activity by inhibiting dehydration. Furthermore, glycerol enhances the molecular ink viscosity, favouring not only the tip coating but also the transference from the tip to the substrate.

Substrate preparation. Substrates used for contact angle measurements as well as for AFM studies were obtained as follows. The $\mathrm{SiO}_{2} / \mathrm{Si}$ substrate was prepared by initially cutting the Si wafers into $0.5 \mathrm{~cm} \times 0.5 \mathrm{~cm}$ pieces. Then, the substrate was washed in an ultrasonic bath for 10 minutes progressively in acetonitrile, ethanol and Milli Q water, and dried by blowing nitrogen gas. $\mathrm{Nb}$ substrates were washed following the same methodology. The $\mu$ SQUID surface was cleaned by carefully washing it with ethanol and acetone, and dried by blowing nitrogen gas before DPN deposition.

Dip-pen nanolithography setup. DPN experiments were performed with a commercial dip-pen writer; NSCRIPTOR ${ }^{\mathrm{TM}}$ DPN $^{\circledR}$ System (NanoInk, Inc., USA). All DPN patterning experiments were carried out under ambient conditions ( $\sim 35 \%$ relative humidity, room temperature). Commercial silicon nitride Type M Probe Arrays (NanoInk, Inc., USA) with a spring constant of $0.5 \mathrm{~N} \cdot \mathrm{m}^{-1}$ were used for the patterning experiments. To coat the tip, a microfluidic ink delivery chip-based system (Inkwell, NanoInk, Inc., USA) was used. The inkwells contained several reservoirs filled with the desired Apoferritin/CoO@Apoferritin solution with a 
micropipette. Afterwards the ink is transferred to a microwell through a microchannel, where the tip is dipped and coated. The coating procedure first involved the addition of a few drops of this ferritin solution into one of the reservoirs of the inkwell chip, and the AFM tip was then coated by dipping it into the microwell that contained such solution.

Dip-pen nanolithography experiments. The experimental parameters used in this work have already been successfully used for the deposition of ferritin proteins bearing different inorganic nanoparticles onto a wide variety of surfaces. ${ }^{[7]}$ The ferritin deposition took place by dispensing femptoliter volumes of the protein solution onto the specific regions of the pick up coils. In all experiments, ferritin patterning was done at a constant humidity of $35 \%$ and ambient temperature. The ferritin nanoarrays were generated by traversing the tip over the surface in the form of the desired pattern, which is previously designed, as already described in the text. The $\mu$ SQUID pick-up loop consists of two rectangular-shaped coils, each of them with dimensions $50 \times 200 \mu \mathrm{m}^{2}$. Such dimensions are larger than those of the piezo scanner that controls the relative movement of the tip with respect to the sample $(90 \mu \mathrm{m} \times 90 \mu \mathrm{m})$. Therefore, to overcome these limitations and cover the full perimeter of both coils, it is necessary to induce, after each patterning writing, an $x-y$ stage translation that relocates the tip over different coil areas separated by more than $90 \mu \mathrm{m} \times 90 \mu \mathrm{m}$. Such translation is controlled by the software and optically controlled with the coupled camera, as can be seen in Figure S6.
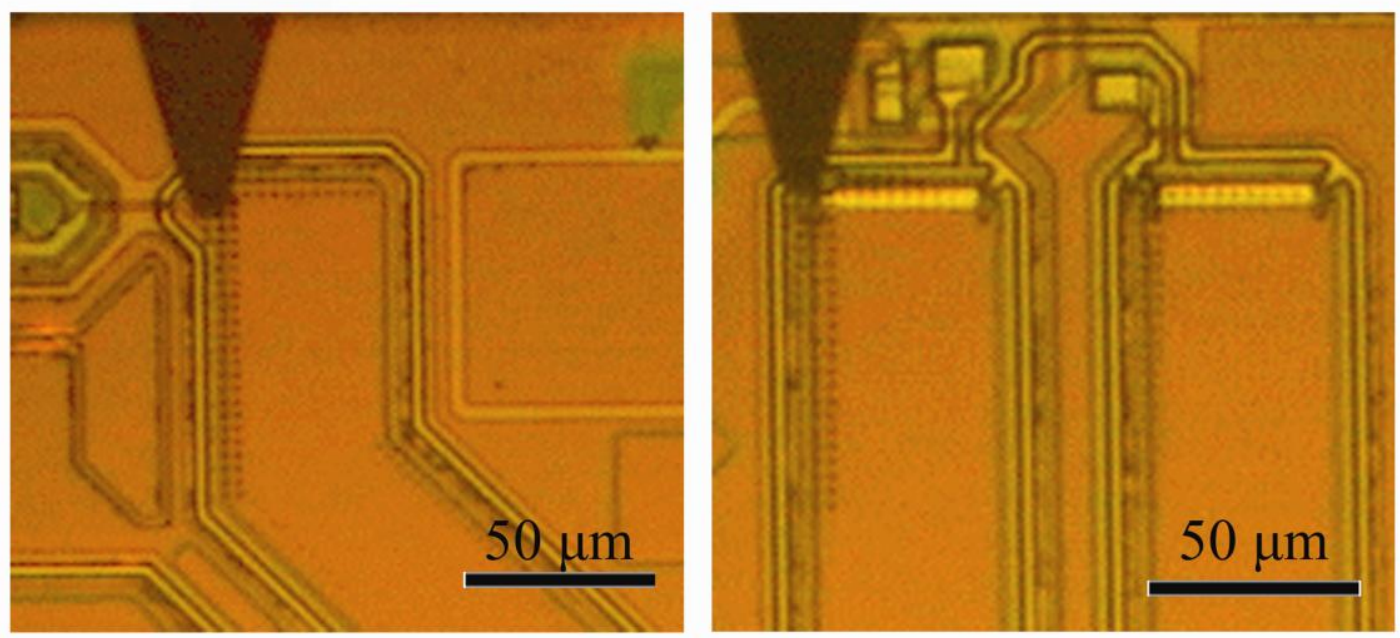

Figure S6. DPN camera images show the writing procedure followed by DPN in order to deposit CoO@Apoferritin particles along the perimeter of the SQUID pick-up loop. 


\section{S6. Bulk magnetization measurements}

The bulk magnetic response of the $\mathrm{CoO} @$ Apoferritin nanoparticles has been characterized with a commercial MPMS magnetometer from Quantum Design over a few milligrams of the sample fixed with Apiezon-N grease on a typical holder. Magnetic measurements down to $1.8 \mathrm{~K}$ confirmed the antiferromagnetic character of the nanoparticles. Antiferromagnetic bulk materials exhibit no net (or negligible) magnetic moment since the magnetization of the two sublattices cancels. However, a small net magnetic moment appears when the size of the crystal is reduced as a consequence of poor crystallinity, surface disorder or spin canting. ${ }^{[11]}$ In the case of the $\mathrm{CoO} @$ Apoferritin nanoparticles the magnetic characterization enables us to quantify this effect giving a net magnetic moment per particle of $\sim 10 \mu_{\mathrm{B}}$. The magnetic characterization down to $\mathrm{mK}$ temperatures (below $1.8 \mathrm{~K}$ ) of the bulk material has been performed by gluing a small quantity of the ferritin-Apiezon mixture $(\approx 1 \mu \mathrm{g})$ on the microsusceptometer pick up coils and cooling it inside the mixing chamber of the dilution refrigerator unit. The excitation magnetic field is produced by applying an input voltage through a room temperature resistance $\left(\mathrm{R}_{\mathrm{ext}}=2 \mathrm{k} \Omega\right)$ using the oscillator generator of a commercial lock-in amplifier. The output voltage is then acquired as a function of frequency using phase-sensitivity lock-in detection. The component in phase with the input voltage is proportional to the real part of the samples susceptibility $\left(\chi^{\prime}\right)$, whereas the out-of-phase component is proportional to the imaginary part $(\chi$ "). The resulting signal is transformed into magnetic units by scaling it with measurements obtained in the commercial magnetometer over a convenient temperature range where both measurements overlap.

The susceptibility data measured on the array and the bulk sample at a frequency of $21 \mathrm{~Hz}$ are compared in Fig. S7a. The two have the same qualitative dependence on temperature above $100 \mathrm{mK}$. However, below this temperature the susceptibility of the array shows a maximum which, as shown in Fig. 3 of the manuscript, depends on frequency. The effect can be ascribed to the better thermalization of the array with the Helium bath, on account of its much larger contact area. The data of the bulk can be made equal to those of the array by shifting them along the temperature axis (Fig. S7b). The temperature shift between the two experiments, estimated in this way, is shown in Fig. S7c. There is a noticeable effect already below $200 \mathrm{mK}$. This analysis suggests that nanopatterning molecular samples, with low thermal conductivities, can provide a good method to investigate their magnetic properties down to the neighbourhood of the absolute zero, when thermalization effects become a crucial issue. 


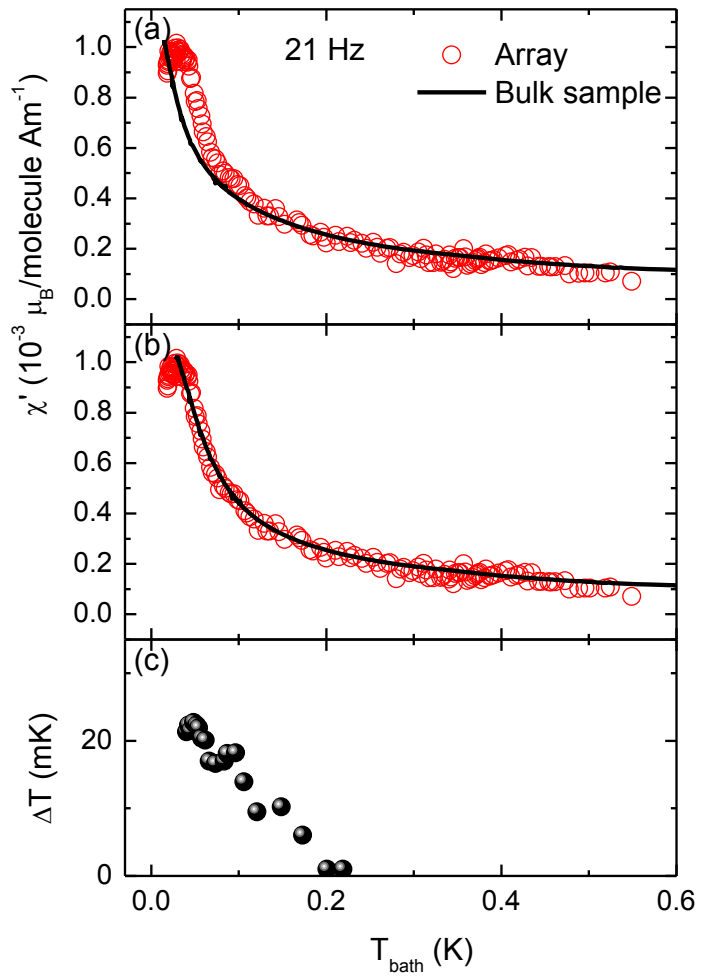

Figure S7. a) Direct comparison of the ac susceptibilities of the Co@apoferritin array deposited by DPN (open dots) and of the bulk sample (solid line) measured at $21 \mathrm{~Hz}$. b) The same data as in a), but in this case the bulk data have been shifted in temperature to match those of the array.

c) Temperature shift between the bulk and array data.

\section{REFERENCES}

[1] D. Drung, C. Aßmann, J. Beyer, A. Kirste, M. Peters and F. Ruede, Th. Schurig, IEEE Trans. Appl. Supercond. 17, 699-704 (2007).

[2] M. J. Martínez-Pérez, J. Sesé, R. Córdoba, F. Luis, D. Drung and T. Schurig, Supercond. Sci. Technol. 22, 125020 (2009).

[3] J. Sesé, F. Lera, A. Camón and C. Rillo, IEEE Trans. Appl. Superconduct. 9, 58-62 (1999).

[4] K. Tsukamoto, M. Iwahori, M., Muraoka and I. Yamashita, Bull. Chem. Soc. Jpn. 78, 2075-2081 (2005).

[5] M. Hassel and H. J. Freund, Surf. Sci. 325, 163 (1995). 
[6] S. A. Chambers, R. F. C. Farrow, S. Maat, M. F. Toney, L. Folks, J. G. Catalano, T. P. Trainor and G. E. Brown, J. Magn. Magn. Mat. 246, 124-139 (2002).

[7] E. Bellido, R. de Miguel, J. Sesé, D. Ruiz-Molina, A. Lostao and D. Maspoch, Scanning 32, 35-41 (2010).

[8] E. Bellido, R. de Miguel, D. Ruiz-Molina, A. Lostao and D. Maspoch, Adv. Mater. 22, 352-355 (2010).

[9] A. K. Panwar, S. K. Barthwal and S. Ray, J. Adhesion Sci. Technol. 17, 1321-1329 (2003).

[10] L. Xu and M. Salmeron in Nano-Surface Chemistry: Studies of Wetting and Capillary Phenomena at Nanometer Scale with Scanning Polarization Force Microscopy, M. Rosoff editor (Marcel Dekker Inc., New York, 2001), chapter 6, pp. 243-288.

[11] L. Néel, C. R. Acad. Sci. 252, 4075-4080 (1961); ibid 253, 9-12 (1961); ibid 253, 1286$1291(1961)$. 